\title{
Identifying Sentiment Influences Provoked by Context Factors - Results from a Data Analytics Procedure Performed on Tweets
}

\author{
Daniel Konadl \\ University of Regensburg \\ daniel.konadl@ur.de
}

\author{
Janik Wörner \\ University of Regensburg \\ janik.woerner@ur.de
}

\author{
Susanne Leist \\ University of Regensburg \\ susanne.leist@ur.de
}

\begin{abstract}
Context factors have lasting impacts on people's sentiments. Exploring impacts that different contexts have on sentiments can be crucial for managing the increasing number of communications companies nowadays maintain with customers via social media channels. To help companies prevent impacts of negative word of mouth, we provide an overview about sentiment-influential contexts for tweets as one kind of social media texts previously discussed within the literature. We collected an overall amount of 358.923.210 tweets and performed analysis to uncover the effects of continents, mobile devices' operating systems $(O S)$ and the combination of both on sentiments expressed within tweets. Our results show remarkable differences for tweets originating from North America and Apple devices, which turned out to be the tweets with the lowest sentiments compared to the other continents and the mobile OS Android.
\end{abstract}

\section{Motivation}

Over the last decade, social media have reached an immense widespread $[1,2]$. In private settings, social media are used for connecting with friends, for communicating with each other, presenting oneself to other users, sharing personal experiences or achieving social standing and reputation within communities [3]. Textual social media (e.g. tweets on Twitter or posts in Facebook) have proven to be a channel for complaint articulation for customers, e.g. about inadequate behaviors of companies resulting from negative experiences with products and services $[4,5]$. Negative perceptions and experiences can prompt consumers to articulate negative sentiments online in written social media [4-7]. As social media texts are widely visible within social media channels, negative sentiments articulated in social media texts can potentially infect huge masses of social media users and provoke negative sentiments [5-7]. Additionally, social media texts containing negative sentiments tend to spread more quickly [8] so that companies have to react fast to prevent damages. Companies reacting adequately and quickly to customers' concerns can turn negative experiences into positive perceptions $[5,9,10]$. Otherwise, negative perceptions can provoke other users to contribute their own negative experiences [5]. This so-called negative word of mouth (nWoM) can lead to the far-reaching dissemination of negative perceptions towards a company in the online as well as in the offline sphere $[5,7]$. Beyond keeping potential customers away from buying companies' products and consuming their services, nWoM can furthermore lead to the termination of existing customer relationships [11-13]. Eventually, churning customers and the absence of new customers lead to decreasing sales [11-13], high costs for acquiring new customers [14] and thereafter even to companies' existence being threatened.

For monitoring the opinions expressed in social media texts, sentiment analysis offers a solution to automatically identify opinion polarities from huge volumes of textual data (cf. [15]). The results of sentiment analysis can inform companies about negative sentiments expressed in social media channels, to make companies try to meet and overcome customers' concerns. While sentiment analysis enables companies to identify negative customers' perceptions identified from the texts' contents, companies require knowledge about the circumstances provoking certain sentiments. In addition to the contents of the texts captured by sentiment analysis tools, there are other factors that influence the sentiments of people.

For example, the days during a week have been shown to predominantly provoking negative sentiments compared to weekends (cf. [16-18]). Furthermore, places related to spare-time activities (e.g. parks and green spaces [19-21]) are associated with more positive sentiments than workplaces where sentiments are on average less positive (e.g. [22]). Companies that are aware of contexts as sentimentinfluential factors can tailor their customer communication according to the effects of contexts. For strengthening the effects of positive messages propa- 
gated, companies can also rely on contexts provoking positive sentiments. Companies aiming at positive Word of Mouth (pWoM) spread (cf. [4, 11, 23]) for evoking positive perceptions towards their products, services or the company itself, should rather communicate related messages e.g. on weekends.

Depending on the locations (e.g. country or continent), customers may be more or less likely expressing negative sentiments within social media posts. To counter the sentiment tendencies prevalent in certain locations (e.g. countries or continents), companies could thereafter adjust their customer communication. We address this research gap and perform analysis to identify the influences mobile devices' operating systems (OS) have on sentiments expressed within tweets as one kind of social media posts. As a second context, we include continents. To address the lack of investigations considering at least two contexts, we perform analysis combining mobile devices' OS and continents as contextual factors.

The rest of the paper is organized as follows: In the next chapter, conceptional basics of social media, word-of-mouth, sentiment analysis and contexts are introduced. Afterwards, within the chapter related work, we give an overview of sentiment-influential context factors and corresponding effects. Subsequently, we describe the steps performed within our investigation and report the results of our analysis. After interpreting and discussing the said results, we draw on implications of our findings. The paper is rounded off with a conclusion including limitations and an outlook on future research.

\section{Conceptional basics}

In literature, the term "social media" is often described as "a group of Internet-based applications that build on the ideological and technological foundations of Web 2.0, and that allow the creation and exchange of User Generated Content (UGC)" ([24], p. 61). UGCs represent "the sum of all ways in which people make use of social media" ([24], p. 61). Social media tools provide users with functionalities to connect with friends, presenting themselves to other users and communicating with each other [3]. Social media posts are suitable to conduct word-of-mouth propaganda and to lead communication that includes personal experiences and opinions about a product, service or promotion $[4,5]$ with consumers, friends, colleagues or other acquaintances [23, 25]. Within these communications, messages as well as therein expressed sentiments are spread [23, 25] and widely noticeable by other users within a social media channel [4-7]. To help companies take notice and control the sentiments expressed within the ever-increasing amount of social media posts, sentiment analysis proposes algorithmic approaches to identify the polarity of texts [15]. In terms of sentiment analysis, there are different approaches, amongst others dictionary- and sentence-based sentiment analysis [15]. When performing dictionary-based sentiment analysis, the sentiment of each entity (e.g. each word) from a text is classified into a positive or negative class using the dictionaries. These dictionaries annotate opinion carrying words, and the sentiment of the whole sentence is determined by considering the sum of the combined scores of all its entities [26].

However, not only the content or the formulation of messages or opinions of customers on products, services or the company itself, but also contexts play an important role in provoking sentiments. Depending on the manifestations of contexts, people experience different sentiments [27]. Context can be defined as "any information that can be used to characterize the situation of an entity" ([28], p. 5) whereby "an entity is a person, place, or object that is considered relevant" ([28], p. 5). Contexts such as time, location or mobile OS as well as combinations of contexts can be investigated as sentiment-influential factors in the offline world. While time describes the temporal contexts (e.g. time of day, day of week, month of year) in which a tweet is posted, location relates to the spatial properties (e.g. county, country, continent) the user is surrounded by, when tweeting. Mobile devices' OS (e.g. Google Android or Apple iOS) responsible for operating essential system functions on mobile devices, are associated with different personality traits [29] and can thus be seen as another sentiment-influential context. Additionally, contexts are as well experienced, while being in the situation of writing social media posts (e.g. tweets). Therefore, contexts also act as sentiment-influential factors within social media spheres (e.g. Twitter).

\section{Related Work}

The idea of observing contextual factors and their influence on sentiments apparent within tweets is not a new one. Efforts have been made to uncover the effects of temporal factors such as time of day [16, $18,30]$ and day of week [16-18, 31] on sentiments within tweets. Tuesdays, Wednesdays and Thursdays have been identified as days characterized by negative emotions [18], with Wednesdays having even been identified as being the most negative days of the week [16]. Throughout the week and towards weekends, the sentiments become more positive, with Fridays and Saturdays being the most positive days [16, 17]. Sundays are associated with peaks in positive sentiment [18]. However, literature also reports de- 
clining sentiments on Sundays [26]. Regarding weekends, the results suggest that both positive and negative sentiments are more present as opposed to the working week [31]. Regarding the times of a day, there are contradictory findings within the literature. While [30] state that the time between 5 a.m. and 6 a.m. is the happiest hour of a day, [18] identified the most negative sentiments for this phase and the most positive sentiments in the evenings. [16] showed that there is a maximum of positive sentiments from 11 a.m. to 1 p.m. as well as from 5 p.m. to 8 p.m.

As for spatial influences, efforts have been made to analyze the effects of different kinds of locations and the impact of their properties on sentiments [16, 17, 19-21, 32-37]. Staying in green spaces such as parks has been identified as inducing positive sentiments within tweets [19-21]. In line with that, [16] identified that how a location is used, influences the sentiments expressed in tweets. Farmland and places associated with public transportation or industry bring up more negative than positive sentiments. Public places are almost equally likely to provoke positive and negative sentiments. Commercial areas tend to generate more positive than negative sentiments [16]. Places where time is shared with friends and family also induce predominantly positive sentiments [17]. The weather prevailing at a location is another sentiment-influential context [33-37]. People are happier when temperatures drop slowly rather than rapidly [35]. Extremely hot and cold temperatures [34], cloudy weather [34], high humidity [34], excessive snowfall [35], hailstorms [35], and extreme weather events such as hurricanes $[33,36]$ or earthquakes [37] have negative influences on the sentiment expressed within tweets.

There is further context information that rely on characteristics of individuals. People with high incomes articulate themselves more positively within tweets as people living in poorer neighborhoods [32, $38,39]$. Older people express more fear compared to middle-aged people whose tweets contain more joyful terms [38]. Neighborhoods with higher proportions of White, Asian and Hispanic populations also share predominantly more joy-related expressions [38]. However, as [39] showed, Hispanic residents can also be associated with less positive and sadder emotions. People with an African background tend to use more dimness-related terms in their tweets [38]. Higher degrees of education shape and higherearning populations share happier and more positive emotions [39]. Tweets that are sent from mobile devices tend to be more negative in terms of expressed sentiments than those that are sent from desktops [40, 41]. As for mobile devices' OS, more positive posts are more likely to stem from blackberry devices [42].
Within the literature, there are already approaches relating to the effects of contexts on sentiments expressed within tweets. There are many investigations concerning the identification of temporal, spatial and person-related contexts as well as corresponding effects provoking positive and negative sentiments. Nevertheless, related work mostly focuses on one context solely or considers them isolated from each other. [16, 17, 19-21, 32-37] have dealt with spatial factors and focused on certain countries (e.g. USA (cf. [16, 17, 19, 21, 32-36]), Haiti (cf. [37]) or Australia (cf. [20])) instead of whole continents. [42] analyzed the influence of mobile OS on sentiments. However, the authors do not match these influences with continents (cf. [42]) as we did within our paper. To address this research gap, we performed analysis that combine mobile devices' OS and continents.

\section{Methodology}

To identify the effects of mobile devices' OS, continents and the combination of both on sentiments expressed within tweets, we aligned our approach to the steps proposed within the text-mining procedure of [43]. As we aim at identifying the effects of contexts on sentiments, we also describe how we proceeded this task. We split our approach (cf. figure 1) into (1) preparing the analysis, (2) conducting the analysis and (3) reporting the results.

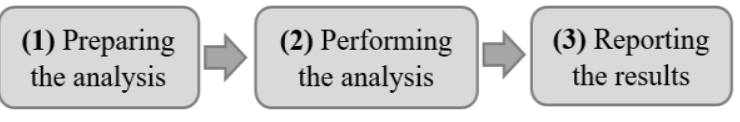

Figure 1: Steps applied within the approach

Within the (1) preparatory steps, we first familiarized with the particularities of tweets. Then, we extracted the tweets and performed exploratory analysis followed by conducting data reduction. Because mobile devices' OS and continents as the context factors to be investigated were not directly provided with the extracted tweets, we had to perform further steps to transform the provided data attribute values to obtain the said context factors. Then, we appended the continents and mobile devices' OS to the corresponding tweets and (2) determined sentiment values for the extracted tweets by applying an existing sentiment analysis approach. As with the continents and mobile devices' OS, sentiment values were appended to the corresponding tweets. Within the next step, summarization techniques are applied to the sentiment values for tweets of the investigated context factors. Hereby, the mean sentiment values for each context factor and 
the share of tweets at a certain sentiment level are determined. The last step (3) is about reporting the results. This includes the results to be presented and interpreted. The obtained results may contradict previous findings so that resolving these conflicts can be necessary. In the next chapters, we describe in more detail how we approached within these three steps.

\section{Preparing the analysis}

\subsection{Particularities of tweets}

As tweets are the research objects within our investigation, we firstly familiarized ourselves with the particularities of Twitter and tweets as the corresponding social media texts. Hereby, Twitter is a microblogging application "stand[ing] halfway between traditional blogs and social networking sites, and are characterized by a high degree of selfpresentation/self-disclosure and a medium to low degree of social presence/media richness" ([44], p. 106). As a specific type of social media, it allows users to "exchange small elements of content such as short sentences, individual images or video links" ([44], p. 106). Tweets are amongst others characterized by their shortness (e.g. [31, 36, 42, 44, 45]). Since November 2017, the maximum number of characters to be used within a tweet is set to 280 (cf. $[46,47])$. This shortness in text length must be taken into consideration when identifying sentiments expressed within tweets [36, 44]. Furthermore, our analysis has to cope with the huge number of users around the world that post tweets (cf. [31, 39, 42, 44, 48]). Therefore, we assume that the methods for processing the tweets need to be fast and performant and must deal with the shortness of tweets to obtain accurate and reliable results [45].

\subsection{Data extraction, data preprocessing and data reduction}

Using Twitter's sampling Application Programmable Interface (API) in the "Spritzer" version, we sampled approximately uniformly from all messages being posted via Twitter in 2019. The data collection procedure resulted in 358.923.210 English tweets in the time range from January $1^{\text {st }}, 2019$ to December $31^{\text {st }}, 2019$. For our investigation, we only collected tweets written in English. To that purpose, we filtered the provided language field to determine only tweets with the value "EN", which indicates that a tweet is written in English language. By this means, we were able to omit the complications of multiple languages (cf. [49]). Further restrictions beyond the language restriction as sampling by only incorporating tweets containing certain hashtags have not been applied.

Fluctuations in collective public emotions and sentiments can occur due to a multitude of competing effects (cf. [50]) and can influence the results of this investigation. By examining a whole year of tweets, seasonal influences and corresponding distortions resulting from deviating levels of sentiments expressed in certain months, such as higher temperature and more positive sentiments in summer months than in winter months, can be omitted. Therefore, because all twelve months of a year are included in our investigation, the influences of the incorporated contexts regarding sentiments are not distorted by a month being not included. As our data collection comprises 358.923.210 tweets, we assume that influences of external events and competing effects are smoothed to a high degree. We further regard the sample as a representative collection of tweets appropriate for our investigations because Twitter's "Spritzer" API provides $1 \%$ of all tweets posted with a maximum margin of error of 0.06 at a confidence level of $99 \%$ [51]. Each tweet delivered by Twitter comes as a JSON (Java Script Object Notation) object that contains tweet text and meta data characterizing both tweets and the situation in which it has been posted. These meta data, such as timestamp, language, source, geolocation and the device used for tweeting (cf. [19]), are logged and provided by Twitter.

Subsequently, we performed exploratory analysis to identify noise and outliers within the obtained data. By this means, we noticed that not every tweet is provided with location or device information. Thus, we agreed on performing analysis concerning influences of location and mobile devices' OS contexts only with tweets containing this context information. Filling up missing values by applying any of the commonly proposed strategies (cf. [52]) could have distorting effects as there were many data instances with missing attributes. To ensure the dataset to be without any redundant tweets, we additionally applied redundancy detection using tweet text and the creation date as the properties of a redundancy. The following steps of data reduction were carried out as part of the transformation of the JSON objects into CSV (Comma Separated Values) files. In this step, we excluded entries that don't match the goals of our investigation. Many of the provided meta data are not necessary for our analysis so that we only included the device field, the self-reported location field and the tweet text to identify sentiments expressed within the tweets. Only a small fraction of the tweets has geolocation coordinates that can be mapped directly to locations in terms of latitude and longitude (cf. [48]). Therefore, we relied on parsing the free- 
response location field that accompanies a tweet. By applying the methods from the Pycountry library (https://pypi.org/project/pycountry/), we mapped the self-reported location information to continents and appended the continent to the corresponding tweet within the CSV files.

\subsection{Selection of methods to be applied}

Identifying the influences of contexts on sentiments expressed within tweets requires a method to assign sentiment values to tweets. Considering the findings from subchapter 5.1., we decided to apply the freely accessible "Valence Aware Dictionary for sEntiment Reasoning" (VADER) approach [45]. VADER was specifically developed for sentiment analysis in microblog-like texts and has achieved remarkable results compared to other sentiment analysis approaches [45]. The approach is also fast enough and can deal with huge numbers of tweets as required for our investigation. Because VADER identifies sentiment values using a built-in sentiment dictionary [45], there is no need for labeled training data. VADER provides a compound sentiment score that combines positive and negative sentiments into one single value. This score can take values in the range of -1 to +1 [45] and is calculated separately for each tweet contained in the data set. Furthermore, we applied summarization measures and decided to calculate mean values in terms of sentiments for all contexts being investigated. As the calculation of mean sentiment values consolidates several sentiment values to one single value, we additionally decided to determine the number of tweets whose sentiments are at certain intervals. This included counting the number of tweets that have certain sentiment values regarding the investigated context factors. In doing so, determining the sentiment intervals is independent from calculating the mean sentiment values.

To test the statistical significance of our findings, we additionally carried out t-tests (cf. [53]). In our case, there are independent samples since one sample selected from one population is not related in any way to the sample from another population [53]. This is because the assignment of tweets and the respective sentiment to a continent and a mobile devices' OS is exclusive and does not consider an assignment to more than one continent or mobile devices' OS. To be applicable, the tests require the dependent variable to be at least interval scaled. We see this requirement fulfilled as the dependent variable (sentiment value) of each tweet is numeric. Additionally, the independent variables (contextual factors) are at least nominalscaled [53]. In the following chapters, every time the term "significant" or "significantly" is used, the dif- ferences in mean sentiment values or proportions within sentiment intervals showed to be significant by the pairwise calculated t-tests.

\section{Performing the analysis}

We observed a high proportion of neutral tweets by filtering for tweets with a sentiment value of " 0 " within the appended sentiment value field. As this huge number of neutral tweets can have remarkable influences on the results, we decided to exclude neutral tweets from further analysis. Therefore, we proceeded with the remaining 245.077.312 tweets being either positive or negative. Then, we filtered the tweets ( $c f$. table 1 ) along with the corresponding sentiment values. For the analysis regarding one context, either continents or devices, we applied one filter criterion. E.g. by filtering the tweets with the value (a) "Europe" or (b) "Android", all tweets originating from (a) Europe (cf. table 1 - IDs 1, 2 and 4) or tweets sent from an Android OS (cf. table 1 - IDs 1 and 4) and their sentiment values are retrieved. When combining the contexts of continents and mobile devices' OS, we simultaneously set two filter criteria. We retrieve e.g. all tweets sent from Android powered devices originating from Europe together with the corresponding sentiment values by filtering with "Android" and "Europe" (cf. table 1 - IDs 1 and 4).

Thereafter, the mean values of the sentiment values regarding the selected singular and combinatorial contexts were calculated and the numbers of occurrences of sentiment values in the respective sentiment intervals were counted. The results of determining the mean sentiment values and the sentiment intervals are more closely described in the next chapter.

Table 1: Example Tweets and Results

\begin{tabular}{|c|c|c|c|}
\hline IDs & \multicolumn{3}{|c|}{ Example Tweets } \\
\hline 1 & \multicolumn{3}{|c|}{$\begin{array}{l}\text { I pray that your August will be full of good news, } \\
\text { positivity and blessings. }\end{array}$} \\
\hline 2 & \multicolumn{3}{|c|}{ Hope you're having a great week. } \\
\hline 3 & \multicolumn{3}{|c|}{ I wish you all the best :-) } \\
\hline 4 & \multicolumn{3}{|c|}{$\begin{array}{l}\text { I have been on hold with you for } 40 \text { minutes and } \\
\text { then the call hangs up. Poor customer service!!! }\end{array}$} \\
\hline$\ldots$ & \multicolumn{3}{|c|}{$\ldots$} \\
\hline \multirow{2}{*}{ IDs } & \multicolumn{3}{|c|}{ Results } \\
\hline & Continent & Mobile OS & Sentiment \\
\hline 1 & Europe & Android & 0.8481 \\
\hline 2 & Europe & Apple & 0.7732 \\
\hline 3 & Asia & Apple & 0.8481 \\
\hline 4 & Europe & Android & -0.4767 \\
\hline$\ldots$ & $\ldots$ & $\ldots$ & $\ldots$ \\
\hline
\end{tabular}




\section{Reporting the results}

\subsection{Results of univariate analysis}

Users of Apple devices turned out to be less positive compared to users of Android devices (cf. table 2). Tweets posted by Android devices have on average higher sentiments than tweets sent by Apple devices. Although the differences for mean sentiment values between Apple and Android users seem to be comparably low in our analysis, they showed to be significant. We additionally determined the number of tweets whose sentiments are at certain intervals (cf. table 4). Regarding the mobile devices' OS, the distributions reflect that $12.89 \%$ of the tweets sent by Apple devices are strongly negative (within [-1; 0.66[), compared to $9.32 \%$ for tweets sent by Android devices. As regards the strongly positive tweets (within [+0.66 to +1]), the proportion of Android tweets is about $5 \%$ higher than the proportion of tweets sent by Apple devices.

In terms of location as the second context, we obtained results for six continents, namely Africa $(A F)$, Asia (AS), Europe (EU), North America (NA), Oceania (OC) and South America (SA). Tweets originating from AS showed to be the most positive, followed by tweets from SA, EU and AF (cf. table 2). Interestingly, the average sentiment values for tweets from OC and NA show significant differences. In addition, we notice that tweets in the range of +0.66 to +1.0 originate most frequently from SA, followed by AS, EU and OC (cf. table 4). Considering the mean sentiment values, it was assumable for NA having a low proportion of tweets within this interval. Based on the mean sentiment value of AF, it is remarkable that tweets are strongly positive (within $[+1 ;+0.66])$ with a proportion of only $23.19 \%$. However, tweets with mean sentiment values between +0.33 and +0.66 occur most often for $\mathrm{AF}$, while the other continents have at least $7.85 \%$ fewer proportions in this interval. When investigating the intervals for negative sentiments, it is significant that NA consistently has the highest proportions, followed by OC. Asia having the highest mean sentiment value, interestingly shows as well comparably high proportions in the strongly negative and the negative intervals (within $[-1 ;-0.66[$ and $[-0.66 ;-0.33[$ ).

Table 2: Sentiments of singular contexts

\begin{tabular}{|c|l|c|}
\hline \multicolumn{2}{|c|}{ Singular Contexts } & Mean Sentiment Values \\
\hline \multirow{4}{*}{ Continents } & Asia & 0.21452 \\
\cline { 2 - 3 } & South America & 0.19716 \\
\cline { 2 - 3 } & Europe & 0.19327 \\
\cline { 2 - 3 } & Africa & 0.18906 \\
\hline
\end{tabular}

\begin{tabular}{|l|l|l|}
\hline & Oceania & 0.16195 \\
\cline { 2 - 3 } & North America & 0.12869 \\
\hline \multirow{2}{*}{$\begin{array}{l}\text { Mobile } \\
\text { OS }\end{array}$} & Android & 0.20179 \\
\cline { 2 - 3 } & Apple & 0.13223 \\
\hline
\end{tabular}

\subsection{Results of bivariate analysis}

The observations of the univariate analysis ( $c f$. tables 2 and 4) are partly reflected within the bivariate analysis (cf. table 3 and 5). Combining each continent with Android gives on average always more positive sentiment values as if the same continents are combined with Apple. Hereby, the highest difference can be observed for Asian tweets, where Android achieves on average significantly higher sentiment values compared to Apple $(\Delta 0.10366)$. South American and African tweets from Android devices are also significantly more positive. Differences observed for NA, EU and OC are however comparably marginal. Tweets posted from AF, EU and OC using Apple devices have consistently higher proportions within the negative sentiment intervals and consistently lower proportions for the positive sentiment intervals (cf. table 5). South American tweets having a sentiment value in the range of $] 0 ;+0.33$ [ occur slightly more often for Apple devices than Android. In Asia, there are also comparably many positive tweets that are sent from Apple devices compared to Android. However, strongly positive tweets (within $[+0.66 ;+1.0]$ ) originate significantly more often from Android devices $(\Delta 9.33 \%)$. The observations for North America are also remarkable, as one would expect to find a higher number of positive tweets from Apple devices because these devices originate from NA. However, NA does not show the expected higher sentiment values due to a possible connection of this continent to the brand Apple that is based there. But Android powered devices predominantly originating from AS provoke more positive sentiment scores for this continent.

Table 3: Sentiments of combined contexts

\begin{tabular}{|l|l|c|}
\hline \multicolumn{2}{|c|}{ Combined Contexts } & Mean Sentiment Values \\
\hline \multirow{4}{*}{ Android } & Asia & 0.25694 \\
\cline { 2 - 3 } & South America & 0.22781 \\
\cline { 2 - 3 } & Africa & 0.21481 \\
\cline { 2 - 3 } & Europe & 0.19987 \\
\cline { 2 - 3 } & Oceania & 0.16436 \\
\cline { 2 - 3 } & North America & 0.13537 \\
\hline \multirow{5}{*}{ Apple } & Europe & 0.17091 \\
\cline { 2 - 3 } & Asia & 0.15328 \\
\cline { 2 - 3 } & Africa & 0.15323 \\
\cline { 2 - 3 } & South America & 0.14674 \\
\cline { 2 - 3 } & Oceania & 0.14323 \\
\cline { 2 - 3 } & North America & 0.10668 \\
\hline
\end{tabular}


Table 4: Proportions of sentiment intervals for continents and mobile devices' OS

\begin{tabular}{|l|c|c|c|c|c|c|}
\hline \multicolumn{1}{|c}{$\begin{array}{c}\text { Singular } \\
\text { Contexts }\end{array}$} & {$[-\mathbf{- 1}-\mathbf{- 0 . 6 6}[$} & {$[-\mathbf{- 0 . 6 6 ; - 0 . 3 3 [}$} & {$[-\mathbf{- 0 . 3 3 ; 0 [}$} & $\mathbf{] 0 ;} \mathbf{0 . 3 3}[$ & {$[\mathbf{0 . 3 3} \mathbf{0 . 6 6 [}$} & {$[\mathbf{0 . 6 6 ;} \mathbf{1 ]}$} \\
\hline Africa & $8.46 \%$ & $13.05 \%$ & $8.94 \%$ & $10.02 \%$ & $36.34 \%$ & $23.19 \%$ \\
\hline Asia & $9.32 \%$ & $14.36 \%$ & $9.65 \%$ & $11.15 \%$ & $28.11 \%$ & $27.41 \%$ \\
\hline North America & $12.03 \%$ & $16.92 \%$ & $10.77 \%$ & $11.41 \%$ & $26.49 \%$ & $22.38 \%$ \\
\hline Europe & $9.02 \%$ & $15.12 \%$ & $10.02 \%$ & $11.63 \%$ & $28.49 \%$ & $25.72 \%$ \\
\hline Oceania & $10.31 \%$ & $15.93 \%$ & $10.61 \%$ & $11.66 \%$ & $27.74 \%$ & $23.75 \%$ \\
\hline South America & $8.12 \%$ & $13.89 \%$ & $8.86 \%$ & $10.28 \%$ & $26.33 \%$ & $32.52 \%$ \\
\hline Android & $9.32 \%$ & $14.55 \%$ & $10.01 \%$ & $10.89 \%$ & $27.57 \%$ & $27.66 \%$ \\
\hline Apple & $12.89 \%$ & $17.21 \%$ & $10.89 \%$ & $11.19 \%$ & $25.80 \%$ & $22.02 \%$ \\
\hline
\end{tabular}

Table 5: Proportions sentiment intervals for the combinations of continents and mobile devices' OS

\begin{tabular}{|c|c|c|c|c|c|c|c|c|c|c|c|c|}
\hline \multirow{3}{*}{$\begin{array}{c}\begin{array}{c}\text { Combined } \\
\text { Contexts }\end{array} \\
\text { Continents } \\
\end{array}$} & \multicolumn{12}{|c|}{ Mobile OS } \\
\hline & \multicolumn{6}{|c|}{ Apple } & \multicolumn{6}{|c|}{ Android } \\
\hline & {$[-1 ;-0.66[$} & {$[-0.66 ;-0.33[$} & {$[-0.33 ; 0[$} & ] $0 ; 0.33[$ & {$[0.33 ; 0.66[$} & {$[0.66 ; 1]$} & {$[-1 ;-0.66[$} & {$[-0.66 ;-0.33[$} & {$[-0.33 ; 0[$} & ] $0 ; 0.33[$ & {$[0.33 ; 0.66[$} & {$[0.66 ; 1]$} \\
\hline Africa & $10.73 \%$ & $16.19 \%$ & $10.50 \%$ & $11.55 \%$ & $27.08 \%$ & $23.95 \%$ & $8.34 \%$ & $14.04 \%$ & $10.19 \%$ & $11.64 \%$ & $28.13 \%$ & $27.66 \%$ \\
\hline Asia & $10.16 \%$ & $16.55 \%$ & $10.68 \%$ & $11.13 \%$ & $29.43 \%$ & $22.05 \%$ & $7.77 \%$ & $13.21 \%$ & $8.80 \%$ & $11.21 \%$ & $27.63 \%$ & $31.38 \%$ \\
\hline North America & $12.96 \%$ & $19.38 \%$ & $11.13 \%$ & $10.81 \%$ & $25.94 \%$ & $19.78 \%$ & $10.71 \%$ & $17.27 \%$ & $10.87 \%$ & $11.01 \%$ & $26.40 \%$ & $23.74 \%$ \\
\hline Europe & $9.42 \%$ & $16.44 \%$ & $10.35 \%$ & $11.42 \%$ & $28.25 \%$ & $24.12 \%$ & $7.98 \%$ & $15.66 \%$ & $10.30 \%$ & $11.64 \%$ & $27.84 \%$ & $26.58 \%$ \\
\hline Oceania & $8.26 \%$ & $18.15 \%$ & $11.75 \%$ & $12.50 \%$ & $27.53 \%$ & $21.81 \%$ & $9.46 \%$ & $16.45 \%$ & $10.58 \%$ & $11.96 \%$ & $27.57 \%$ & $23.98 \%$ \\
\hline South America & $10.26 \%$ & $16.82 \%$ & $10.76 \%$ & $12.35 \%$ & $28.90 \%$ & $20.91 \%$ & $7.79 \%$ & $14.29 \%$ & $9.37 \%$ & $11.34 \%$ & $29.14 \%$ & $28.07 \%$ \\
\hline
\end{tabular}




\subsection{Interpretation and implications}

Actions, decisions and sentiments expressed within social media texts are influenced by the contexts in which people act. Certain manifestations of contexts thereby provoke more likely positive or negative sentiments. While reacting is only possible after certain conditions have already occurred, including contexts into decision-making enables companies to perform preventive actions. They can benefit by adapting the way they communicate within certain markets by aligning to the sentiments provoked by contexts. E.g. as customers from certain countries or continents may be more or less likely expressing negative sentiments within social media posts, companies could thereafter adjust their customer communication to counter sentiment tendencies prevalent there. Regarding the results of our analysis, companies should especially adapt messages for customers originating from NA, and those North Americans that send tweets from their Apple devices. Although the contexts NA and Apple provoke negative sentiments, the combination of NA and Apple devices turned out to provoke the most negative sentiments according to our results (cf. tables 2 and 3). Therefore, we suppose that there is the most potential for this combination when companies want to include contexts into decision-making within the activities and tasks of customer communication. By providing tailored contents and messages for these customers, the negative attitudes caused by the corresponding context factors of NA (continent) and Apple (mobile devices' OS) could be countered. Instead, when companies intentionally aim at provoking $\mathrm{pWoM}$, they should better concentrate on AS and Android users.

Our findings retrieved for the mobile devices' OS are supported by the findings of [29] who report their results from a psychological investigation. Users of Apple devices are associated with more negative traits. They are perceived as less honest, less humble and are considered to manipulate others more often to gain personal advantages [29]. Hereby, the results of [42] are contradicting the findings of [29] and our results. In this work, tweets sent from Blackberry devices are associated with more positive sentiments compared to tweets sent from Apple or Android devices (cf. [42]). As the data set of [42] comprises the time range of May $1^{\text {st }}, 2012$ to April $30^{\text {th }}, 2014$, we assume that this data no longer reflect the current situation of mobile devices being used. The observed differences of Android users being more positive within our results could therefore be justified by Blackberry users that switched to Android devices. The market share of Blackberry has continuously dropped so that there are nowadays predominantly two major mobile OS, Android and Apple's iOS, that dominate the mobile devices market [54].

\section{Conclusion}

Our paper provides an overview about sentimentinfluential contexts within tweets, which is followed by the identification of the influences of continents, mobile devices' OS and the combination of both. Our approach is structured into preparatory steps, the execution of the analysis and the reporting of the corresponding results. The results of our analysis have implications for the management of customer communications within social media channels because companies strongly build on social media to foster the external communication with customers (e.g. [55, 56]). NWoM expressed by disappointed or angry customers within social media channels (e.g. Twitter or Facebook) has the potential to negatively impact the perception of (potential) customers towards a company (cf. [5-7, 11-13]). As customers reporting negative experiences within Twitter await replies within one to three hours, companies have to react quickly [10] or even better take preventive actions. Hereby, the contexts inducing negative sentiments can support the corresponding decision-making. In addition, we see contributions of contexts for companies intentionally aiming at provoking $\mathrm{pWoM}$. Companies that use Twitter for customer communication benefit most from our results and findings about sentiment-inducing contexts due to the required higher reaction speed [10]. Our results can support this task as our investigation is based on tweets as research objects. Companies apply Facebook as well as a customer communication channel where customers also articulate negative perceptions and experiences (cf. $[5,6,10])$. Therefore, we recommend as a possible step for future research to give an overview of sentiment-influential contexts and corresponding effects within Facebook posts.

The paper on hand is however not free of limitations. First, the analysis performed in our investigation was only performed with the VADER sentiment approach. Secondly, our results can be seen rather as initial findings derived from a data analytics procedure. Therefore, it is a necessary step in future research to deduce more sound recommendations from our results. Thirdly, the results of our work are based on the tweets of the year 2019. For this reason, we propose to apply the described analysis procedure to the data of previous years and to figure out observed similarities and differences regarding the influences of context factors. 


\section{References}

[1] Dickey, I.J., and Lewis, W.F., "The evolution (revolution) of social media and social networking as a necessary topic in the marketing curriculum: a case for integrating social media into marketing classes", Advances in Marketing: Embracing Challenges and Change-A Global Perspective. 2010.

[2] Chaffey, D., "Global social media research summary 2016", Smart Insights: Social Media Marketing. 2016.

[3] Kietzmann, J.H., Hermkens, K., McCarthy, I.P., and Silvestre, B.S., "Social media? Get serious! Understanding the functional building blocks of social media", Business horizons. 54(3), 2011, pp. 241-251.

[4] Jansen, B.J., Zhang, M., Sobel, K., and Chowdury, A., "Twitter power: Tweets as electronic word of mouth", Journal of the American society for information science and technology. 60(11), 2009, pp. 2169-2188.

[5] Grégoire, Y., Salle, A., and Tripp, T.M., "Managing social media crises with your customers: The good, the bad, and the ugly", Business Horizons. 58(2), 2015, pp. 173-182.

[6] Einwiller, S.A., and Steilen, S., "Handling complaints on social network sites-An analysis of complaints and complaint responses on Facebook and Twitter pages of large US companies", Public Relations Review. 41(2), 2015, pp. 195-204.

[7] Pfeffer, J., Zorbach, T., and Carley, K.M., "Understanding online firestorms: Negative word-ofmouth dynamics in social media networks", Journal of Marketing Communications. 20(1-2), 2014, pp. 117128.

[8] Naveed, N., Gottron, T., Kunegis, J., and Alhadi, A.C., "Bad news travel fast: A content-based analysis of interestingness on twitter", in Proceedings of the 3rd international web science conference, 2011.

[9] Joireman, J., Grégoire, Y., Devezer, B., and Tripp, T.M., "When do customers offer firms a "second chance" following a double deviation? The impact of inferred firm motives on customer revenge and reconciliation", Journal of Retailing. 89(3), 2013, pp. 315-337.

[10] Istanbulluoglu, D., "Complaint handling on social media: The impact of multiple response times on consumer satisfaction", Computers in Human Behavior. 74, 2017, pp. 72-82.

[11] Chevalier, J.A., and Mayzlin, D., "The effect of word of mouth on sales: Online book reviews", Journal of marketing research. 43(3), 2006, pp. 345-354.

[12] Mizerski, R.W., "An attribution explanation of the disproportionate influence of unfavorable information", Journal of Consumer Research. 9(3), 1982, pp. 301-310.

[13] Kim, S.J., Wang, R.J.-H., Maslowska, E., and Malthouse, E.C., "'Understanding a fury in your words": The effects of posting and viewing electronic negative word-of-mouth on purchase behaviors", Computers in Human Behavior. 54, 2016, pp. 511-521.

[14] Greenberg, P., "CRM at the speed of light: Capturing and keeping customers in Internet real time": Elsevier. 2001.
[15] Liu, B., "Sentiment analysis and opinion mining": Morgan \& Claypool Publishers. 2012.

[16] Cao, X., MacNaughton, P., Deng, Z., Yin, J., Zhang, X., and Allen, J.G., "Using twitter to better understand the spatiotemporal patterns of public sentiment: a case study in Massachusetts, USA", International journal of environmental research and public health. 15(2), 2018.

[17] Hu, T., She, B., Duan, L., Yue, H., and Clunis, J., "A Systematic Spatial and Temporal Sentiment Analysis on Geo-Tweets", IEEE Access. 8, 2019, pp. 86588667.

[18] Golder, S.A., and Macy, M.W., "Diurnal and seasonal mood vary with work, sleep, and daylength across diverse cultures", Science. 333(6051), 2011, pp. 18781881.

[19] Plunz, R.A., Zhou, Y., Vintimilla, M.I.C., Mckeown, K., Yu, T., Uguccioni, L., and Sutto, M.P., "Twitter sentiment in New York City parks as measure of wellbeing", Landscape and urban planning. 189, 2019, pp. 235-246.

[20] Lim, K.H., Lee, K.E., Kendal, D., Rashidi, L., Naghizade, E., Winter, S., and Vasardani, M., "The grass is greener on the other side: Understanding the effects of green spaces on Twitter user sentiments", in Companion Proceedings of the The Web Conference 2018.

[21] Schwartz, A.J., Dodds, P.S., O'Neil-Dunne, J.P., Danforth, C.M., and Ricketts, T.H., "Visitors to urban greenspace have higher sentiment and lower negativity on Twitter", People and Nature. 1(4), 2019, pp. 476485 .

[22] Sandstrom, G.M., Lathia, N., Mascolo, C., and Rentfrow, P.J., "Putting mood in context: Using smartphones to examine how people feel in different locations", Journal of Research in Personality. 69, 2017, pp. 96-101.

[23] Kaplan, A.M., and Haenlein, M., "Two hearts in threequarter time: How to waltz the social media/viral marketing dance", Business horizons. 54(3), 2011, pp. 253-263.

[24] Kaplan, A.M., and Haenlein, M., "Users of the world, unite! The challenges and opportunities of Social Media", Business horizons. 53(1), 2010, pp. 59-68.

[25] Hennig-Thurau, T., Wiertz, C., and Feldhaus, F., "Does Twitter matter? The impact of microblogging word of mouth on consumers' adoption of new movies", Journal of the Academy of Marketing Science. 43(3), 2015, pp. 375-394.

[26] Kundi, F.M., Ahmad, S., Khan, A., and Asghar, M.Z., "Detection and scoring of internet slangs for sentiment analysis using SentiWordNet", Life Science Journal. 11(9), 2014, pp. 66-72.

[27] Mehrabian, A., " An approach to environmental psychology", 1974, Cambridge, MA: MIT Press, 1974.

[28] Dey, A.K., "Understanding and using context", Personal and ubiquitous computing. 5(1), 2001, pp. 47.

[29] Shaw, H., Ellis, D.A., Kendrick, L.-R., Ziegler, F., and Wiseman, R., "Predicting smartphone operating system from personality and individual differences", 
Cyberpsychology, Behavior, and Social Networking. 19(12), 2016, pp. 727-732.

[30] Dodds, P.S., Harris, K.D., Kloumann, I.M., Bliss, C.A., and Danforth, C.M., "Temporal patterns of happiness and information in a global social network: Hedonometrics and Twitter", PloS one. 6(12), 2011.

[31] Wang, W., Hernandez, I., Newman, D.A., He, J., and Bian, J., "Twitter analysis: Studying US weekly trends in work stress and emotion", Applied Psychology. 65(2), 2016, pp. 355-378.

[32] Mitchell, L., Frank, M.R., Harris, K.D., Dodds, P.S., and Danforth, C.M., "The geography of happiness: Connecting twitter sentiment and expression, demographics, and objective characteristics of place", PloS one. 8(5), 2013.

[33] Mandel, B., Culotta, A., Boulahanis, J., Stark, D., Lewis, B., and Rodrigue, J., "A demographic analysis of online sentiment during hurricane irene", in Proceedings of the second workshop on language in social media, 2012: Association for Computational Linguistics.

[34] Baylis, P., Obradovich, N., Kryvasheyeu, Y., Chen, H., Coviello, L., Moro, E., Cebrian, M., and Fowler, J.H., "Weather impacts expressed sentiment", PloS one. 13(4), 2018.

[35] Li, J., Wang, X., and Hovy, E., "What a nasty day: Exploring mood-weather relationship from twitter", in proceedings of the 23rd ACM International Conference on Conference on Information and Knowledge Management, 2014.

[36] Neppalli, V.K., Caragea, C., Squicciarini, A., Tapia, A., and Stehle, S., "Sentiment analysis during Hurricane Sandy in emergency response", International journal of disaster risk reduction. 21, 2017, pp. 213-222.

[37] Oh, O., Kwon, K.H., and Rao, H.R., "An Exploration of Social Media in Extreme Events: Rumor Theory and Twitter during the Haiti Earthquake 2010", in ICIS, 2010.

[38] Lin, Y.-R., "Assessing sentiment segregation in urban communities", in Proceedings of the 2014 international conference on Social Computing, 2014.

[39] Lerman, K., Arora, M., Gallegos, L., Kumaraguru, P., and Garcia, D., "Emotions, demographics and sociability in Twitter interactions", in Tenth International AAAI Conference on Web and Social Media, 2016.

[40] Murthy, D., Bowman, S., Gross, A.J., and McGarry, M., "Do we tweet differently from our mobile devices? A study of language differences on mobile and webbased Twitter platforms", Journal of Communication. 65(5), 2015, pp. 816-837.

[41] Groshek, J., and Cutino, C., "Meaner on mobile: Incivility and impoliteness in communicating contentious politics on sociotechnical networks", Social Media+ Society. 2(4), 2016.
[42] Cruz-Albrecht, L., Xu, J., Ni, K.-Y., and Lu, T.-C., "Characterizing Regional and Behavioral Device Variations Across the Twitter Timeline: A Longitudinal Study", in Proceedings of the 2017 ACM on Web Science Conference, 2017.

[43] Aggarwal, C.C., and Zhai, C., "Mining text data": Springer Science \& Business Media. 2012.

[44] Kaplan, A.M., and Haenlein, M., "The early bird catches the news: Nine things you should know about micro-blogging", Business horizons. 54(2), 2011, pp. 105-113.

[45] Hutto, C.J., and Gilbert, E., "Vader: A parsimonious rule-based model for sentiment analysis of social media text", in Eighth international AAAI conference on weblogs and social media, 2014.

[46] Newton, C., "Twitter just doubled the character limit for tweets to 280", The Verge. 2017.

[47] Busby, M., "Twitter to introduce expanded 280character tweets for all its users", The Guardian. 2017.

[48] Schwartz, H.A., Eichstaedt, J.C., Kern, M.L., Dziurzynski, L., Lucas, R.E., Agrawal, M., Park, G.J., Lakshmikanth, S.K., Jha, S., and Seligman, M.E., "Characterizing geographic variation in well-being using tweets", in Seventh International AAAI Conference on Weblogs and Social Media, 2013.

[49] Thelwall, M., Buckley, K., and Paltoglou, G., "Sentiment in Twitter events", Journal of the American Society for Information Science and Technology. 62(2), 2011, pp. 406-418.

[50] Lansdall-Welfare, T., Dzogang, F., and Cristianini, N., "Change-point analysis of the public mood in UK Twitter during the Brexit referendum", in 2016 IEEE 16th International Conference on Data Mining Workshops (ICDMW), 2016: IEEE.

[51] Gerlitz, C., and Rieder, B., "Mining one percent of Twitter: Collections, baselines, sampling", M/C Journal. 16(2), 2013.

[52] Bramer, M., "Principles of data mining", Vol. 180: Springer. 2007.

[53] Sakai, T., "Two sample t-tests for ir evaluation: Student or welch?", in Proceedings of the 39th International ACM SIGIR conference on Research and Development in Information Retrieval, 2016.

[54] Statista. "Marktanteile der führenden mobilen Betriebssysteme an der Internetnutzung mit Mobiltelefonen weltweit von September 2009 bis Mai 2020", visited on 06.07.2020, available from: https://de.statista.com/statistik/daten/studie/184335/um frage/marktanteil-der-mobilen-betriebssystemeweltweit-seit-2009/.

[55] Stobbe, A., "Enterprise 2.0-Wie Unternehmen das Web 2.0 für sich nutzen. Deutsche Bank Research", Economics. 78, 2010, pp. 1-16.

[56] Heidemann, J., Klier, M., and Probst, F., "Online social networks: A survey of a global phenomenon", Computer networks. 56(18), 2012, pp. 3866-3878. 